\title{
Nutritional management after hepatopancreatobiliary surgery
}

\author{
Liming Chen ${ }^{1}$, Xiaowei Chen ${ }^{2}, \mathrm{Gu} \mathrm{Li}^{3}$ \\ ${ }^{1}$ Department of Hepatobiliary and Pancreatic Surgery, The First Affiliated Hospital, Zhejiang University School of Medicine, Hangzhou, China; \\ ${ }^{2}$ Department of Surgery, People's Hospital of Jingning She Autonomous County, Lishui, China; ${ }^{3}$ Department of Neurosurgery, The First Affiliated \\ Hospital, Zhejiang University School of Medicine, Hangzhou, China \\ Correspondence to: Liming Chen. Department of Hepatobiliary and Pancreatic Surgery, The First Affiliated Hospital, Zhejiang University School of \\ Medicine, 79 Qingchun Road, Hangzhou 310003, China. Email: 1192010@zju.edu.cn.
}

Submitted Feb 10, 2021. Accepted for publication Mar 10, 2021.

doi: 10.21037/hbsn-2021-10

View this article at: http://dx.doi.org/10.21037/hbsn-2021-10

Since Wilmore and Kehlet first introduced the concepts of fast track protocols and enhanced recovery after surgery (ERAS) in 2001, a growing amount of attention has been paid to clinical research (1). ERAS encompasses multidisciplinary programs in nursing and anesthesia, nutrition and infusion therapy, and minimally invasive surgery. More than 400 related studies have been completed in the past 2 years after the introduction of postoperative care in the ERAS manual. Nutrition is particularly important in enhanced postoperative recovery, as it encourages early postoperative feeding. Malnutrition is a common problem for hospitalized patients, and 30-50\% of hospitalized patients have malnutrition issues (2). Malnutrition affects prognosis and the quality of life of the patients. Therefore, proper nutritional assistance can improve the patient's own immunity, and reduce the incidence of infection and postoperative complications.

Normal liver tissue has amazing regeneration ability. However, huge changes in biochemistry and metabolism after major hepatectomy may bring serious consequences and even threaten lives. If the nutrients are not supplemented properly, a series of serious consequences, such as jaundice, hepatic ascites, and hepatic failure, are likely to occur (3). Most liver resections minimize the incidence of postoperative metabolic problems by improving preoperative nutritional status and providing appropriate postoperative albumin and carbohydrate support. Appropriate management is necessary for postoperative metabolism and promoting liver recovery and regeneration. Additionally, patients undergoing liver resection are more likely to have significantly lower blood glucose levels, while patients undergoing total hepatectomy and liver transplantation have severe hypoglycemia. In any case, it is wise to rule out the danger of hypoglycemia. Standard operation involves monitoring blood glucose and continuing intravenous infusion of $10 \%$ glucose solution for at least a few days during the first phase or before supplying sufficient carbohydrates by oral feeding. Often following oral feeding of carbohydrates, blood sugar may be elevated for a few weeks. Considering that the liver is the site where albumin is synthesized, severe hypoalbuminemia will occur to a significant degree unless the route of feeding is replaced by parenteral administration. Albumin supplementation takes about a week, and by the end of the third postoperative week, the albumin level has basically returned to normal. Several studies $(4,5)$ have pointed out that patients with liver resection should choose a diet rich in branched-chain amino acids in the early postoperative period. In addition, a high-fat diet based on medium-chain triglycerides and supplemented with fatty acids can also be used.

Biliary tract surgery, such as bile duct repair, bile duct stone removal, and other operations, have a greater impact on liver function; thus, patients need to be supplemented with sufficient water after surgery to maintain acid-base balance. In the early postoperative period, high-energy nutritional support should be given intravenously, but fat intake should be restricted. With the gradual recovery of diet, parenteral nutrition supplementation can be gradually reduced after $2-3$ weeks and finally stopped. The supply of bile salt preparations needs particular attention in patients with biliary obstruction and external biliary fistula. Considering the physiological role of bile or bile acid is to emulsify fat and promote the absorption of fat, activate protease and lipase, and promote the absorption of calcium, iron, and some vitamins, the supplementation of bile salt 
preparations is particularly important for the postoperative rehabilitation of biliary surgery patients $(6,7)$.

Many studies (8-10) have reported the influence of the prognostic nutritional index (PNI) on the postoperative efficacy of pancreatic surgery patients. Kanda et al. (8) analyzed the prognosis of 268 pancreatic cancer patients undergoing pancreatectomy. Multivariate analysis showed that lower preoperative PNI (not low albumin) was an independent prognostic factor leading to lower survival rates. Indeed, a significant association between lower preoperative albumin concentration and PNI and postoperative complications has been found. Sato et al. (9) analyzed the experience of 44 patients who had undergone distal pancreatectomy. The researchers noticed that the rapid reduction of postoperative PNI was related to the higher incidence of postoperative pancreatic fistula. The prognosis of pancreatic cancer is another disease closely related to postoperative nutritional management. With the insufficient exocrine and endocrine function of the pancreas, nutritional problems and a high complication rate associated with pancreatectomy are the most severe problems. Recognizing these problems and proposing appropriate solutions can effectively reduce morbidity and mortality. Because pancreatic tumors usually progress to areas other than the pancreas, the resection rate is very low, and, even with total pancreatectomy, survival rates are still much lower. Several methods of nutritional support have been devised thus far, among which oral feeding of nutritional supplements is the least invasive. However, for patients who cannot tolerate oral feeding, the intake of nutrients is inadequate, as it is for those patients with delayed gastric emptying after surgery. Total parenteral nutrition provides sufficient and complete nutritional needs. However, total parenteral nutrition may involve several potential complications. The delivery of high glucose loads requires sufficient insulin, and thus hyperglycemia, metabolic acidosis, and fluid excess may occur without careful monitoring. In contrast, since nutrients are directly delivered to the stomach, duodenum or jejunum, enteral nutrition is more "physiological" solution. Enteral nutrition stimulates the release of pancreaticobiliary secretion, and these luminal nutrients promote metabolism, regulate hormone levels in the gastrointestinal tract, and maintain normal gastrointestinal contraction and blood flow (11). For patients with mild acute pancreatitis, oral feeding must be restored as soon as possible. The beginning of eating resumption does not depend on the normalization of lipase; rather, it may depend on the patient: they can eat when they feel hungry. Reports indicate that patients requesting food usually react positively to a very wide and varied diet, which can include a nonspecified, light, ordinary diet, with or without fat restriction. However, for patients with chronic pancreatitis, especially those with alcohol addiction, conditions of vitamin, electrolyte, and other nutrient deficiencies may arise. It is unclear whether this deficiency is related to the low baseline level caused by chronic alcohol abuse or the rapid loss caused by the acute outbreak of chronic pancreatitis; regardless, deficiencies of calcium, magnesium, zinc, vitamin B1, and folic acid are the most universal $(12,13)$. The comprehensive awareness management of chronic pancreatitis should start with the management of pancreatic enzymes. The ultimate purpose of nutritional management is to increase body weight, increase fat and protein absorption, and reduce the occurrence of steatorrhea sequelae. For a few patients, a diet of high carbohydrates and high protein and a certain degree of fat restriction is required. Medium-chain triglycerides can be used as a source of extra calories. It is critical to control stomach acid, reduce intestinal hyperactivity, and reduce complications of biliary stones. Finally, alcohol intake must be prohibited.

Due to their peculiarities, hepatobiliary, biliary, and pancreatic diseases-or even more malignant hepatopancreatobiliary tumors-can greatly impact the metabolic system, with most afflicted patients experiencing malnutrition after surgery. Malnutrition not only affects the patient's postoperative recovery, but also increases the probability of disease-related complications. For hepatopancreatobiliary patients, reasonable enteral or parenteral nutrition support can significantly improve the nutritional status of the patients after surgery, prevent nutrient deficiency, and improve patient immunity. Moreover, proper nutritional support can effectively reduce the incidence of postoperative infections, reduce the occurrence of various complications, and improve prognosis, thereby promoting the recovery of patients.

\section{Acknowledgments}

Funding: This study was supported by a grant from the National Key Research and Development Program of China (no. 2018YFC2001804).

\section{Footnote}

Provenance and Peer Review: This article was commissioned 
by the editorial office of Hepatobiliary Surgery and Nutrition. The article did not undergo external peer review.

Conflicts of Interest: All authors have completed the ICMJE uniform disclosure form (available at https://hbsn. amegroups.com/article/view/10.21037/hbsn-2021-10/coif). This study was only supported by a grant from the National Key Research and Development Program of China. The authors have no other conflicts of interest to declare.

Ethical Statement: The authors are accountable for all aspects of the work in ensuring that questions related to the accuracy or integrity of any part of the work are appropriately investigated and resolved.

Open Access Statement: This is an Open Access article distributed in accordance with the Creative Commons Attribution-NonCommercial-NoDerivs 4.0 International License (CC BY-NC-ND 4.0), which permits the noncommercial replication and distribution of the article with the strict proviso that no changes or edits are made and the original work is properly cited (including links to both the formal publication through the relevant DOI and the license). See: https://creativecommons.org/licenses/by-nc-nd/4.0/.

\section{References}

1. Wilmore DW, Kehlet H. Management of patients in fast track surgery. BMJ 2001;322:473-6.

2. La Torre M, Ziparo V, Nigri G, et al. Malnutrition and pancreatic surgery: prevalence and outcomes. J Surg Oncol 2013;107:702-8.

3. Masuda T, Shirabe K, Yoshiya S, et al. Nutrition support and infections associated with hepatic resection and liver transplantation in patients with chronic liver disease.

Cite this article as: Chen L, Chen X, Li G. Nutritional management after hepatopancreatobiliary surgery. HepatoBiliary Surg Nutr 2021;10(2):273-275. doi: 10.21037/ hbsn-2021-10
JPEN J Parenter Enteral Nutr 2013;37:318-26.

4. Sun Y, Yang Z, Tan H. Perioperative nutritional support and fluid therapy in patients with liver diseases. Hepatobiliary Surg Nutr 2014;3:140-8.

5. Okabayashi T, Iyoki $M$, Sugimoto T, et al. Oral supplementation with carbohydrate- and branched-chain amino acid-enriched nutrients improves postoperative quality of life in patients undergoing hepatic resection. Amino Acids 2011;40:1213-20.

6. Wendel D, Mortensen M, Harmeson A, et al. Resolving malnutrition with parenteral nutrition before liver transplant in biliary atresia. J Pediatr Gastroenterol Nutr 2018;66:212-17.

7. Ravindranath A, Sen Sarma M, Yachha SK. Bile acid synthetic defects: Simplified approach in a nutshell. Hepatobiliary Pancreat Dis Int 2020;19:80-4.

8. Kanda M, Fujii T, Kodera Y, et al. Nutritional predictors of postoperative outcome in pancreatic cancer. Br J Surg 2011;98:268-74.

9. Sato N, Mori Y, Minagawa N, et al. Rapid postoperative reduction in prognostic nutrition index is associated with the development of pancreatic fistula following distal pancreatectomy. Pancreatology 2014;14:216-20.

10. Mahendran R, Tewari M, Dixit VK, et al. Enhanced recovery after surgery protocol enhances early postoperative recovery after pancreaticoduodenectomy. Hepatobiliary Pancreat Dis Int 2019;18:188-93.

11. Gianotti L, Besselink MG, Sandini M, et al. Nutritional support and therapy in pancreatic surgery: A position paper of the International Study Group on Pancreatic Surgery (ISGPS). Surgery 2018;164:1035-48.

12. O'Brien SJ, Omer E. Chronic pancreatitis and nutrition therapy. Nutr Clin Pract 2019;34:S13-26.

13. Grant JP. Nutritional support in acute and chronic pancreatitis. Surg Clin North Am 2011;91:805-20. 